\title{
Short Communication \\ Ki 67 is a major, but not the sole determinant of Oncotype Dx recurrence score
}

\author{
S Sahebjam', R Aloyz', ${ }^{1,3}$ D Pilavdzic ${ }^{2}$, M-L Brisson², C Ferrario', N Bouganim', V Cohen', WH Miller Jr', \\ and LC Panasci*,1,3 \\ 'Department of Medical Oncology, Jewish General Hospital, McGill University, 3755 Côte Ste-Catherine Road, Montreal, QC H3T IE2, Canada; \\ ${ }^{2}$ Department of Pathology, Jewish General Hospital, MCGill University, Montreal, QC H3T IE2, Canada; ${ }^{3}$ Segal Cancer Center, Lady Davis Institute, \\ Jewish General Hospital, Montreal, QC H3T IE2, Canada
}

\begin{abstract}
BACKGROUND: Immunohistological assessment of Ki 67 expression is less expensive than Oncotype Dx, which is currently used to identify patients with lymph node-negative breast cancer, who will benefit from adjuvant chemotherapy.

METHODS: The relationship of immunohistologically measured Ki 67 to Oncotype DX recurrence score (RS) was examined in 53 cases of TI -2 N0 MO (oestrogen receptor-positive, HER2/neu negative) breast cancer.

RESULTS: There was a strong linear correlation between Ki 67 value and the Oncotype Dx RS. All patients in the low Ki 67 group (Ki 67 of $\leqslant 10 \%$ ) had Oncotype Dx RSs of low or intermediate risk. The vast majority of patients (93.8\%) in the high-Ki 67 group (Ki $67 \geqslant 25 \%$ ) had oncotype RSs of high or intermediate risk.

CONCLUSION: Ki 67 proliferation value is a major, but not the sole determinant of Oncotype Dx score.

British Journal of Cancer (20II) I 05, 1342-1345. doi:I0.1038/bjc.2011.402 www.bjcancer.com

Published online 4 October 2011

(C) 201 I Cancer Research UK
\end{abstract}

Keywords: Ki 67 expression; immunohistochemistry; Oncotype Dx

Ki 67 protein is found in proliferating cells. It is present in the nuclei of cells in G1, S, G2, and M phases of the cell cycle. Ki 67 protein levels are low during G1- and early S-phase, and gradually increase to reach a maximum during mitosis. Therefore, Ki 67 protein expression can be a useful marker of cell proliferation (Urruticoechea et al, 2005).

Multiple studies have shown an association of Ki 67 expression with prognosis and response to systemic treatments in both the neoadjuvant and adjuvant settings. High-Ki 67 expression has been associated with an increased risk of breast cancer recurrence and cancer death (Lee et al, 1997; Colleoni et al, 2004; Kronqvist et al, 2004; Penault-Llorca et al, 2009). Tumours with high Ki 67 before neoadjuvant chemotherapy have a higher rate of pathologic response, and lower $\mathrm{Ki} 67$ after neoadjuvant chemotherapy is associated with favourable disease-free survival (DFS; Nishimura et al, 2010; Yerushalmi et al, 2010). At least two studies have suggested that patients with high-Ki 67 tumours may benefit more from adjuvant chemotherapy and endocrine therapy (Viale et al, 2008a, b; Penault-Llorca et al, 2009).

The Oncotype Dx gene test (Genomic Health, Redwood City, CA, USA) is a commercially available reverse transcriptase PCR assay (RT-PCR) of 21 genes, which uses a specific algorithm to calculate the recurrence score (RS) for oestrogen receptor (ER)-positive breast cancers. On the basis of RS, patients are considered low risk $(\mathrm{RS}<18)$, intermediate risk $(18 \leqslant \mathrm{RS}<31)$, or high risk $(\mathrm{RS} \geqslant 31)$. Although different cancer-related genes including ER and HER-2

*Correspondence: Dr LC Panasci; E-mail: Ipanasci@hotmail.com Received 24 June 2011; revised 9 September 2011; accepted 13 September 201 I; published online 4 October 201 I expression are included in calculating the RS, five proliferation genes (Ki 67, STK 15, Survivin, CCNB 1, and MYBL 2) are heavily weighted and especially important in this calculation (Paik et al, 2004). This test is currently used for predicting the risk of recurrence in lymph node-negative ER-positive breast cancers, and is considered by the National Comprehensive Cancer Network guidelines as an option to help decision-making in this group of patients. Unfortunately, the high cost of this multigene assay limits its' use in daily practice in many countries.

The aim of this study was to compare the correlation of immunohistologically measured $\mathrm{Ki} 67$ protein expression with the Oncotype Dx RS in patients with lymph node-negative $\mathrm{ER} /$ progesterone receptor (PR)-positive, HER-2-negative breast cancers.

\section{MATERIALS AND METHODS}

With approval from the Institutional Research Ethics Committee, we analysed 53 cases of T1-2 N0 M0 (ER/PR-positive, HER-2negative) breast cancer treated in the Jewish General Hospital, Montreal, Canada. These cases were chosen randomly from a pool of patients with early-stage breast cancer, who had Oncotype Dx analysis of their tumour. Pathology reports were reviewed and histological type, tumour size, Nottingham grade, perineural invasion status, lymphovascular invasion status, invasive tumour necrosis, ER levels, and PR levels were recorded. ER and PR levels were recorded as per the Allred Score (Harvey et al, 1999). All tumours were HER-2 negative, using immunohistochemistry (IHC) or fluorescent in situ hybridisation. Sentinel lymph node sampling data were available in all patients and no lymph node 
involvement was reported. The Oncotype Dx RS results were provided by Genomic Health test reports. Patients were stratified to three risk groups as per Oncotype RS: low risk $(\mathrm{RS}<18)$, intermediate risk $(18 \leqslant \mathrm{RS}<31)$, and high risk ( $\mathrm{RS} \geqslant 31)$ (Paik et al, 2004).

Ki 67 expression was examined by IHC of formalin-fixed deparaffinised tissue, using prediluted rabbit monoclonal antibody against human Ki 67 (Clone 30-9, Ventana, Tucson, AZ, USA) at a concentration of $2 \mu \mathrm{g} \mathrm{ml}^{-1}$. Slides were stained on automated immunostainer Benchmark XT from Ventana, using the iView DAB Detection Kit. The same tissue blocks were used for both Oncotype Dx testing and Ki 67 immunostaining. Results were assessed without the use of an image analysis system. The fraction of positive cells (in percentage) with definite nuclear immunostaining, including mild, moderate, and strong was counted. The representative fields were chosen at low magnification and included at least two areas at the most cellular edges of tumour, and one area in the centre. The number of cells counted at highpower magnification varied depending on distribution of $\mathrm{Ki} 67$ immunopositive staining. For cases with even distribution, the $\mathrm{Ki}$ 67 staining was determined with 400-600 tumoural cells, but in cases with uneven distribution, up to 2500 tumoural cells were counted. The stained slides were evaluated by two of the authors when the distribution of Ki-67-positive tumoural cells was uneven, or when the percentage of immunoreactivity was near the cut-off points. Patients were divided into low-risk (Ki $67<10 \%$ ), intermediate-risk $(10 \% \leqslant \mathrm{Ki} 67<25 \%)$, and high-risk group $(\mathrm{Ki} 67 \geqslant 25 \%)$ on the basis of the expression of Ki 67 .

Ki $67<10 \%$ was considered low, based on a cut-off point used by Kronqvist et al (2004) and Breast International Group Trial 1-98 (Viale et al, 2008a, b). However, as there were other studies that used $\mathrm{Ki} 67>20 \%$ as their cut-off point, we decided to consider Ki $67 \geqslant 25 \%$ as high (Colleoni et al, 2004; Viale et al, 2008a,b). By doing so, we could study the group of patients with $\mathrm{Ki} 67$ values falling between these two numbers (Ki 67 intermediate group).

The SPSS version 19 (Chicago, IL, USA) was used for statistical analysis. Linear regression, univariate analysis, multivariate analysis, and partial correlation analysis were performed.

\section{RESULTS}

The pathologic characteristics of patients are presented in Table 1.

The median Ki 67 value was $17.3 \%$ (range $2-90 \%$ ). The median Oncotype RS was 18 (range 7-60). There was a strong linear correlation between Ki 67 expression and Oncotype RS (correlation coefficient $=0.73, P$-value $<0.001$; Figure 1 ).

There was also a significant correlation between Nottingham grade and Oncotype RS on univariate analysis (correlation coefficient $=0.52, P$-value $<0.001)$. The correlation of Ki 67 and Oncotype RS remained robust (correlation coefficient $=0.6$, $P$-value $<0.001$ ), even after controlling for the effect of Nottingham grade by using partial correlation analysis and multivariate analysis. On the other hand, there was no significant correlation between Nottingham grade and Oncotype RS when the effect of Ki 67 was controlled (correlation coefficient $=0.064, P$-value $=0.65$ ). This suggests that the correlation found between Nottingham grade and Oncotype RS on univariate analysis was most likely due to the effect of Ki 67.

We also analysed the correlation between other histopathological characteristics of tumour with Oncotype RS. Previously, Flanagan et al (2008) reported significant correlation between nuclear grade, mitotic count, ER score, and PR score . In our study, there was a weak but significant correlation between nuclear grade and Oncotype RS (correlation coefficient $=0.39, P$-value $=$ $0.005)$. This correlation was weaker but still significant, when the effect of $\mathrm{Ki} 67$ was controlled by using partial correlation analysis (correlation coefficient $=0.32, P$-value $=0.047$ ). There was
Table I Pathological characteristics of tumours

\begin{tabular}{|c|c|}
\hline & Number (\%) \\
\hline \multicolumn{2}{|l|}{ Size } \\
\hline$\leqslant 1 \mathrm{~cm}$ & $6(11.3)$ \\
\hline$>1 \mathrm{~cm}$ & $47(88.7)$ \\
\hline \multicolumn{2}{|l|}{ Histology } \\
\hline Ductal & $48(90.6)$ \\
\hline Lobular & $5(9.4)$ \\
\hline \multicolumn{2}{|l|}{ Nottingham grade } \\
\hline 1 & $15(28.3)$ \\
\hline 2 & $29(54.7)$ \\
\hline 3 & $9(17)$ \\
\hline \multicolumn{2}{|l|}{ Ki 67} \\
\hline Low $(<10)$ & $16(30.2)$ \\
\hline Intermediate $(10 \leqslant$ Ki $67<25)$ & $21(39.6)$ \\
\hline High $(\mathrm{Ki} 67 \geqslant 25)$ & $16(30.2)$ \\
\hline \multicolumn{2}{|l|}{ ER level (Allred score) } \\
\hline Negative $(<3)$ & $0(0)$ \\
\hline Weak (3-4) & I (1.9) \\
\hline Strong $(\geqslant 5)$ & 52 \\
\hline \multicolumn{2}{|l|}{ PR level (Allred score) } \\
\hline Negative $(<3)$ & $6(11.3)$ \\
\hline Weak (3-4) & $9(17)$ \\
\hline Strong $(\geqslant 5)$ & $38(71.7)$ \\
\hline \multicolumn{2}{|l|}{ Oncotype Dx RS category } \\
\hline Low $(\mathrm{RS} \leqslant 17)$ & $25(47.2)$ \\
\hline Intermediate $(18 \leqslant R S \leqslant 30)$ & $20(37.7)$ \\
\hline High $(R S \geqslant 31)$ & $8(\mid 5.1)$ \\
\hline
\end{tabular}

Abbreviations: $E R=$ oestrogen receptor, $\mathrm{PR}=$ progesterone receptor, $\mathrm{RS}=$ recurrence score.

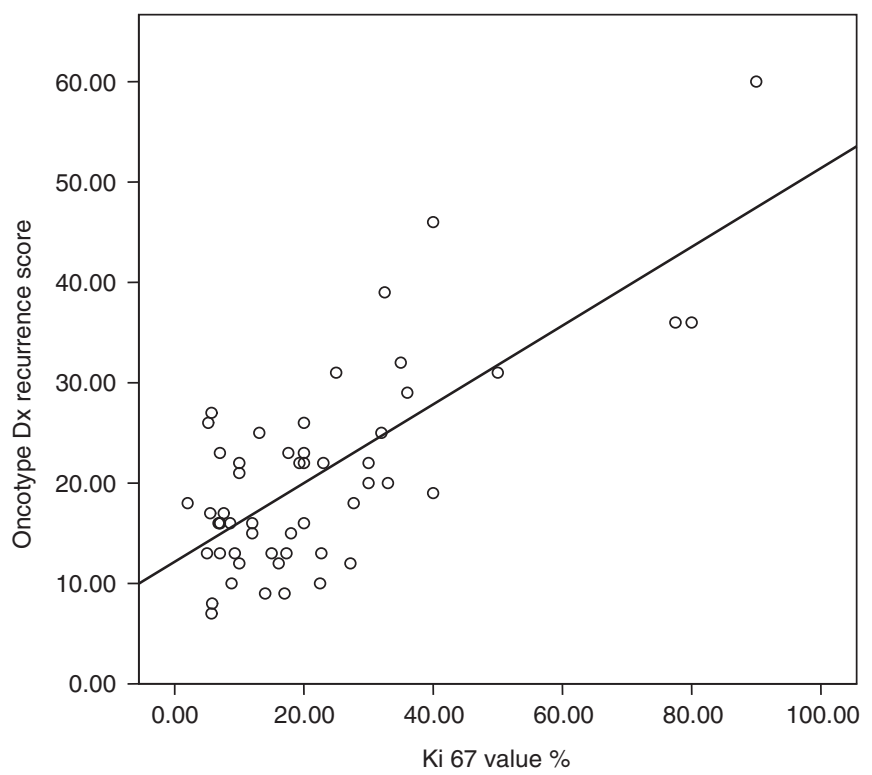

Correlation coefficient $=0.73, P$-value $<0.001$

Figure I Correlation of Oncotype DX RS with Ki 67 value. Correlation coefficient $=0.73, P$-value $<0.001$

a significant correlation between mitosis score (measured as part of Nottingham scoring) and Oncotype RS in univariate analysis (correlation coefficient $=0.39, P$-value $=0.005)$. This correlation 
Table 2 Distribution of cases by Ki 67 value and Oncotype Dx RS

\begin{tabular}{lccc}
\hline & $\begin{array}{c}\text { Low-oncotype } \\
\text { RS (\%) }\end{array}$ & $\begin{array}{c}\text { Intermediate- } \\
\text { oncotype RS (\%) }\end{array}$ & $\begin{array}{c}\text { High-oncotype } \\
\text { RS (\%) }\end{array}$ \\
\hline Low Ki 67 & I $(75)$ & $4(25)$ & $0(0)$ \\
Intermediate Ki 67 & $12(57.1)$ & $9(42.9)$ & $0(0)$ \\
High Ki 67 & I (6.25) & $7(43.85)$ & $8(50)$ \\
\hline
\end{tabular}

Abbreviation: $\mathrm{RS}=$ recurrence score.

was not significant when the effect of $\mathrm{Ki} 67$ was controlled in multivariate analysis and partial analysis (correlation coefficient $=0.054, P$-value $=0.714$ ).

In previous studies, lower expression of PR has been associated with higher Oncotype RS (Flanagan et al, 2008; Tang et al, 2010). Tang et al (2010) demonstrated that among ER-positive tumours, PR-poor tumours had significantly higher Oncotype RS. Our results were consistent with these findings. ER/PR expression had a significant inverse correlation with Oncotype RS, but did not affect the correlation between Ki 67 and Oncotype RS. Lower expression of PR was associated with higher Oncotype RS (correlation coefficient $=-0.56, P$-value $<0.001$ ), which was independent from the effect of ER expression. The combination of ER/PR expression and $\mathrm{Ki} 67$ had a very strong correlation with Oncotype RS (correlation coefficient $=0.84,(0.75-0.93) P$-value $<0.001)$.

There was no significant correlation between perineural invasion, lymphovascular invasion, invasive tumour necrosis, and Oncotype RS on multivariate analysis.

Most patients (93.85\%) in the high-Ki 67 group (Ki $67 \geqslant 25 \%$ ) had Oncotype RS of high or intermediate risk. All patients in lowKi 67 group (Ki 67 of $<10 \%$ ) had RS of low or intermediate risk (Table 2).

\section{DISCUSSION}

Oncotype Dx testing provides valuable prognostic and predictive information in patients with early-stage breast cancers. Unfortunately, the high price limits the accessibility of this test to all patients. Hence, there has been increasing interest to find simple pathology tests, which can help predict the recurrence of disease. As $K i 67$ is one of the proliferation genes assessed routinely by IHC in different malignancies, several investigators have studied its prognostic and predictive value in different stages of breast cancer treatment. High-Ki 67 expression detected by IHC has been reported as the strongest individual prognostic factor of breast cancer death or recurrence in patients with T1 N0 M0 disease (Kronqvist et al, 2004). Ki 67 positivity in more than $10 \%$ of cancer cells increased the odds of breast cancer death or relapse by 11 -fold (Kronqvist et al, 2004). In another study, the 4-year DFS was lower for patients with T1 N0 M0 breast cancer and Ki 67 value $\geqslant 20 \%$, compared with T1 N0 M0 breast cancer and Ki $67<20 \%$; 93.3\% vs $99.2 \%$, respectively (Colleoni et al, 2004).

$\mathrm{Ki} 67$ value has also been considered as a strong predictive factor for effectiveness of neoadjuvant and adjuvant systemic therapy. In a study by Nishimura et al (2010), pathological response after neoadjuvant chemotherapy was significantly associated with $\mathrm{Ki} 67$ values. A higher pathological complete response rate was found in patients with higher Ki 67 values, and there was no pathological response in patients with Ki 67 less than $25 \%$. Ki 67 after neoadjuvant treatment was predictive of DFS. The lower Ki $67(<12 \%)$ was associated with favourable DFS (Nishimura et al, 2010). Expression of Ki 67 in ER-positive breast cancer patients has been associated with benefit from docetaxel treatment in the adjuvant setting; 5-year DFS was higher in patients with ER-positive/Ki 67-positive tumours (84\%) vs ERpositive/Ki 67-negative tumours (81\%) when they were treated with adjuvant docetaxel (Penault-Llorca et al, 2009). Greater benefit of adjuvant endocrine therapy with Letrozole $v s$ Tamoxifen has been observed in patients with Ki 67 value $>10 \%$ (Viale et al, 2008a, b).

$\mathrm{Ki} 67$ has been also found to be highly effective in dividing ER-positive tumours into luminal A and luminal B subtypes (Hugh et al, 2009).

One of the limitations of the use of $\mathrm{Ki} 67$ value is the lack of a standardised scoring system, which should be addressed, as multiple studies, including ours, have underlined the potential use of this test in decision-making.

Our study reveals a strong correlation between $\mathrm{Ki} 67$ value and Oncotype DX RS, especially in tumours with Ki 67 value $\geqslant 25 \%$. Our findings strengthen two recent publications by Gwin et al (2009) and Allison et al (2011). Gwin et al (2009) studied the association between Ki 67 value and Oncotype Dx RS in a series of 32 patients with lymph node-negative ER/PR-positive, HER-2negative breast cancer. Although no correlation analysis was presented in this article, overall association was found between $\mathrm{Ki}$ 67 value and Oncotype RS in most cases. They also noticed unexpectedly high-Ki 67 value in patients with low RS. One of the limitations of the study was the fact that none of the 32 patients had high RS. On the basis of these findings, the authors suggested the combination of Oncotype DX and Ki 67 values for identifying tumours with a high potential of recurrence (Gwin et al, 2009). In addition, recently, Ki 67 was significantly associated with Oncotype RS, Nottingham grade, and angiolymphatic invasion (Williams et al, 2011).

Previously, Flanagan et al (2008) had shown a significant correlation between Nottingham grade and Oncotype RS (correlation coefficient $=0.59, P<0.01$ ). In a recently published article by Allison et al (2011), the correlation of $\mathrm{Ki} 67$ and routine pathological parameters with Oncotype RS was studied. In this study, Nottingham grade had the strongest overall association with Oncotype RS and was used in combination with the PR level to divide cases into different subgroups.

A unique finding in our study is the fact that the correlation between Nottingham score and Oncotype RS disappeared when the effect of Ki 67 value was controlled. Our findings suggest that Ki 67 is the major driver of the correlation found between Nottingham grade and Oncotype RS.

We also found that the likelihood of a tumour with $\mathrm{Ki} 67 \geqslant 25 \%$ having a high or intermediate Oncotype RS is $>90 \%$, and these patients may be the group that benefit the most from adjuvant chemotherapy.

In summary, our data suggests that $\mathrm{Ki} 67$ is the major but not the sole determinant of Oncotype RS. It will be of great interest to study an immunopanel consisting of Ki 67 with other proliferation markers measured by Oncotype Dx, that is, STK 15, Survivin, CCNB1, and MYBL2.

\section{REFERENCES}

Allison KH, Kandalaft PL, Sitlani CM, Dintzis SM, Gown AM (2011) Routine pathologic parameters can predict Oncotype DX(TM) recurrence scores in subsets of ER positive patients: who does not always need testing? Breast Cancer Res Treat; e-pub ahead of print 3 March 2011; doi:10.1007/s10549-011-1416-3
Colleoni M, Rotmensz N, Peruzzotti G, Maisonneuve P, Viale G, Renne G, Casadio C, Veronesi P, Intra M, Torrisi R, Goldhirsch A (2004) Minimal and small size invasive breast cancer with no axillary lymph node involvement: the need for tailored adjuvant therapies. Ann Oncol 15: $1633-1639$ 
Flanagan MB, Dabbs DJ, Brufsky AM, Beriwal S, Bhargava R (2008) Histopathologic variables predict Oncotype DX recurrence score. Mod Pathol 21: $1255-1261$

Gwin K, Pinto M, Tavassoli FA (2009) Complementary value of the Ki-67 proliferation index to the oncotype DX recurrence score. Int J Surg Pathol 17: $303-310$

Harvey JM, Clark GM, Osborne CK, Allred DC (1999) Estrogen receptor status by immunohistochemistry is superior to the ligand-binding assay for predicting response to adjuvant endocrine therapy in breast cancer. J Clin Oncol 17: 1474-1481

Hugh J, Hanson J, Cheang MC, Nielsen TO, Perou CM, Dumontet C, Reed J, Krajewska M, Treilleux I, Rupin M, Magherini E, Mackey J, Martin M, Vogel C (2009) Breast cancer subtypes and response to docetaxel in node-positive breast cancer: use of an immunohistochemical definition in the BCIRG 001 trial. J Clin Oncol 27: 1168-1176

Kronqvist P, Kuopio T, Nykänen M, Helenius H, Anttinen J, Klemi P, South-Western Finland Breast Group (2004) Predicting aggressive outcome in T1N0M0 breast cancer. Br J Cancer 91: 277-281

Lee AK, Loda M, Mackarem G, Bosari S, DeLellis RA, Heatley GJ, Hughes K (1997) Lymph node negative invasive breast carcinoma 1 centimeter or less in size (T1a,bN0M0): clinicopathologic features and outcome. Cancer 79: $761-771$

Nishimura R, Osako T, Okumura Y, Hayashi M, Arima N (2010) Clinical significance of Ki-67 in neoadjuvant chemotherapy for primary breast cancer as a predictor for chemosensitivity and for prognosis. Breast Cancer 17: 269-275

Paik S, Shak S, Tang G, Kim C, Baker J, Cronin M, Baehner FL, Walker MG, Watson D, Park T, Hiller W, Fisher ER, Wickerham DL, Bryant J, Wolmark N (2004) A multigene assay to predict recurrence of tamoxifen-treated, node-negative breast cancer. $N$ Engl J Med 27: $2817-2826$

Penault-Llorca F, André F, Sagan C, Lacroix-Triki M, Denoux Y, Verriele V, Jacquemier J, Baranzelli MC, Bibeau F, Antoine M, Lagarde N, Martin
AL, Asselain B, Roché H (2009) Ki67 expression and docetaxel efficacy in patients with estrogen receptor-positive breast cancer. J Clin Oncol 27: $2809-2815$

Tang P, Wang J, Hicks DG, Wang X, Schiffhauer L, McMahon L, Yang Q, Shayne M, Huston A, Skinner KA, Griggs J, Lyman G (2010) A lower Allred score for progesterone receptor is strongly associated with a higher recurrence score of 21-gene assay in breast cancer. Cancer Invest 28: $978-982$

Urruticoechea A, Smith IE, Dowsett M (2005) Proliferation marker Ki-67 in early breast cancer. J Clin Oncol 23: $7212-7220$

Viale G, Giobbie-Hurder A, Regan MM, Coates AS, Mastropasqua MG, Dell'Orto P, Maiorano E, MacGrogan G, Braye SG, Ohlschlegel C, Neven P, Orosz Z, Olszewski WP, Knox F, Thürlimann B, Price KN, Castiglione-Gertsch M, Gelber RD, Gusterson BA, Goldhirsch A, Breast International Group Trial 1-98 (2008a) Prognostic and predictive value of centrally reviewed Ki-67 labeling index in postmenopausal women with endocrine-responsive breast cancer: results from Breast International Group Trial 1-98 comparing adjuvant tamoxifen with letrozole. J Clin Oncol 26: $5569-5575$

Viale G, Regan MM, Mastropasqua MG, Maffini F, Maiorano E, Colleoni M, Price KN, Golouh R, Perin T, Brown RW, Kovács A, Pillay K, Ohlschlegel C, Gusterson BA, Castiglione-Gertsch M, Gelber RD, Goldhirsch A, Coates AS, International Breast Cancer Study Group (2008b) Predictive value of tumor Ki-67 expression in two randomized trials of adjuvant chemoendocrine therapy for node-negative breast cancer. J Natl Cancer Inst 100: $207-212$

Williams DJ, Cohen C, Darrow M, Page AJ, Chastain B, Adams AL (2011) Proliferation (Ki-67 and phosphohistone H3) and Oncotype DX recurrence score in estrogen receptor-positive breast cancer. Appl Immunohistochem Mol Morphol 19: 431 - 436

Yerushalmi R, Woods R, Ravdin PM, Hayes MM, Gelmon KA (2010) Ki67 in breast cancer: prognostic and predictive potential. Lancet Oncol 11: $174-183$ 